\title{
BMJ Open Effects of lumbar extensor muscle strengthening and neuromuscular control retraining on disability in patients with chronic low back pain: a protocol for a randomised controlled trial
}

Joshua Brodie Farragher, ${ }^{\oplus}$ Adrian Pranata, ${ }^{2}$ Gavin Williams, ${ }^{1}$ Doa El-Ansary, ${ }^{2}$ Selina M Parry, ${ }^{1}$ Jessica Kasza, ${ }^{3}$ Adam Bryant ${ }^{1}$

To cite: Farragher JB, Pranata A, Williams G, et al. Effects of lumbar extensor muscle strengthening and neuromuscular control retraining on disability in patients with chronic low back pain: a protocol for a randomised controlled trial. BMJ Open 2019;9:e028259. doi:10.1136/ bmjopen-2018-028259

- Prepublication history for this paper is available online To view these files, please visit the journal online (http://dx.doi org/10.1136/bmjopen-2018028259).

Received 06 December 2018 Revised 17 June 2019 Accepted 11 July 2019

\section{Check for updates}

(c) Author(s) (or their employer(s)) 2019. Re-use permitted under CC BY-NC. No commercial re-use. See rights and permissions. Published by BMJ.

For numbered affiliations see end of article.

\section{Correspondence to} Joshua Brodie Farragher; jfarragher@student.unimelb. edu.au

\section{ABSTRACT}

Introduction Chronic low back pain (CLBP) is the leading cause of disability worldwide. However, there is no consensus in the literature regarding optimal management. Exercise intervention is the most widely used treatment as it likely influences contributing factors such as physical and psychological. Literature evaluating the effects of exercise on CLBP is often generalised, non-specific and employs inconsistent outcome measures. Moreover, the mechanisms behind exerciserelated improvements are poorly understood. Recently, research has emerged identifying associations between neuromuscular-biomechanical impairments and CLBPrelated disability. This information can be used as the basis for more specific and, potentially more efficacious exercise interventions for CLBP patients.

Methods and analysis Ninety-four participants (including both males and females) with CLBP aged 18-65 who present for treatment to a Melbournebased private physiotherapy practice will be recruited and randomised into one of two treatment groups. Following baseline assessment, participants will be randomly allocated to receive either: (i) strengthening exercises in combination with lumbar force accuracy training exercises or (ii) strengthening exercises alone. Participants will attend exercise sessions twice a week for 12 weeks, with assessments conducted at baseline, midway (ie, 6 weeks into the trial) and at trial completion. All exercise interventions will be supervised by a qualified physiotherapist trained in the intervention protocol. The primary outcome will be functional disability measured using the 0swestry Disability Index. Other psychosocial and mechanistic parameters will also be measured

Ethics and dissemination This study was given approval by the University of Melbourne Behavioural and Social Sciences Human Ethics Sub-Committee on 8 August 2017, reference number 1749845 . Results of the randomised controlled trial will be published in peerreviewed journals.

Trial registration number ACTRN12618000894291.

\section{Strengths and limitations of this study}

- Both the assessor and participants are blinded to their group allocation. The treating therapists are not blinded.

- This is the first randomised controlled trial to investigate the efficacy of neuromuscular control exercise specifically targeting lumbar muscle force accuracy.

- The novel assessment of biomechanical parameters such as whole body kinematics during lifting and variable force accuracy of the lumbar extensors.

- There is no long-term follow-up.

- The trial protocol is dependent on specific equipment for replication or generalisation.

\section{INTRODUCTION}

Chronic low back pain (CLBP) is the leading cause of disability worldwide. ${ }^{1}$ In 2015 , $\sim 540$ million $^{2}$ or $7.3 \%$ of the world's population had CLBP-related disability. ${ }^{3}$ The ability to carry out daily activities is an important outcome for patients with CLBP. ${ }^{4}$ Patient-reported outcome measures are ideal tools for measuring disability. The most frequently utilised condition-specific disability measure for people with CLBP is the Oswestry Disability Index (ODI). ${ }^{56}$

CLBP is a complex and multifactorial condition with both disability and pain linked to physical, neurophysiological, psychological and social factors. ${ }^{7}$ Physical and neurophysiological factors that have been found to associate with CLBP-related disability include lumbar extension strength deficits $^{8}$ and decreased lumbar multifidus (LM) size. $^{9}$ Moreover, novel measures of lumbar extensor force accuracy and control $^{10}$ and 
lifting-related inter-joint coordination ${ }^{11}{ }^{12}$ have been associated with CLBP-related disability.

Diminished movement coordination between the trunk and the lower limbs during lifting has been associated with the development of CLBP. ${ }^{13}{ }^{14}$ Moreover, people with higher CLBP-related disability have demonstrated kinematic and kinetic mal-adaptations during lifting. ${ }^{12} 15$ Indeed, a positive weak correlation $(\mathrm{r}=0.30, \mathrm{p}=0.048)$ has been identified between trunk and hip movement coordination and self-reported disability. ${ }^{11}$ Furthermore, functional tasks such as lifting often use sub-maximal force of the lumbar extensor muscles ${ }^{16}$; thus, there may be functional relevance in examining sub-maximal lumbar extension force accuracy. In support, a recently developed lumbar extensor force accuracy test demonstrated that it may predict up to $19 \%$ of the variance in CLBP-related disability. ${ }^{10}$

While the abovementioned factors are more associated with CLBP-related disability, muscle morphology of the lumbar spine has a moderate positive relationship with pain duration $(\mathrm{F}=6.34, \mathrm{p}=0.016) .{ }^{17}$ No association has been established with self-reported CLBP-related disability $^{17}{ }^{18}$; however, these studies participants had minimal disability, which may not be representative of a more disabled population. LM is a significant contributor to control and segmental stabilisation of the lumbar spine. ${ }^{19}{ }^{20}$ In CLBP patients, a number of imaging studies have reported altered morphology and decreased size of the LM compared with matched healthy individuals. ${ }^{921-23}$ Moreover, people with unilateral CLBP display significantly reduced size of the LM on the symptomatic side of the lumbar spine compared with the asymptomatic side. ${ }^{9}$

In addition to lumbar muscle morphology, architectural differences in lumbar muscle tissue have been demonstrated in CLBP patients. MRI studies have demonstrated that CLBP patients have increased fat infiltration and decrease in cross-sectional area of their erector spinae and LM muscles. ${ }^{24}$ Fat infiltration, a sign of lumbar muscle deconditioning, may increase the echogenicity (ie, 'whiteness') on real-time ultrasound (US) images. ${ }^{25}$ The erector spinae muscles, in particular the more muscular lateral bands that connect the ilium, lumbar and lower thoracic region known as the iliocostalis lumborum (IL), are important in maintaining static and dynamic posture (ie, during lifting). ${ }^{26-28}$ Considering this, future studies should evaluate morphological and architectural parameters of the IL in addition to the LM and how these muscles respond to resistance training in people with CLBP.

Awareness of the psychosocial factors associated with CLBP has increased dramatically in recent years. ${ }^{29}$ In particular, there have been a number of studies investigating the relationship between kinesiophobia and pain self-efficacy among CLBP patients. ${ }^{30-34}$ The Tampa Scale for Kinesiophobia (TSK) and Pain Self-Efficacy Questionnaire (PSEQ) should be considered when investigating this population, as they are associated with CLBP-related chronicity. ${ }^{35}$ Thus, these measures may help explain the mechanism(s) for improvements in CLBP-related disability. ${ }^{7}$

Despite the numerous studies investigating the effects of various types of exercise on disability in people with CLBP, ${ }^{36-38}$ the underlying mechanisms behind how and why people do/don't improve remain unknown. Previous CLBP-related interventional studies have focused on improving some of the abovementioned parameters. ${ }^{373940}$ However, no previous randomised controlled trial (RCT) has investigated force accuracy and lifting-related interjoint coordination that, of the biomechanical-neuromuscular factors, have a demonstrated stronger association with CLBP-related disability. ${ }^{10-12}$

\section{Objectives}

The primary objective of this study is to determine in patients with CLBP whether the addition of lumbar neuromuscular control retraining exercises to a 12-week programme of general and back-specific strengthening exercises result in significant improvements in disability compared with 12 weeks of general and back-specific strengthening alone.

The secondary objective of this study is to: investigate the neuromuscular, biomechanical and psychosocial mechanisms in which neuromuscular control retraining and muscle strengthening may influence CLBP-related disability.

\section{Hypothesis}

It is hypothesised that CLBP participants receiving neuromuscular control exercise targeted at improving lumbar extensor force accuracy in addition to strengthening exercise will demonstrate a greater reduction in percieved disability compared with those only receiving strengthening exercises.

\section{METHODS}

\section{Design and setting}

The design is a two-arm randomised-controlled trial, which will be conducted at a single primary care physiotherapy centre in Melbourne, Australia and will be conducted over approximately 15 months (between July 2018 and October 2019). A total of 94 participants with CLBP will be recruited from a Melbourne-based private physiotherapy clinic. New patients presenting to the clinic with CLBP will be screened by a physiotherapist for eligibility into the study. Participants will be randomly allocated to receive either: (i) 12 weeks of global and isolated strengthening exercises or; (ii) 12 weeks of global and isolated strengthening exercises and a neuromuscular control exercise. The trial design is outlined in figure 1.

\section{Recruitment and participants}

A physiotherapist will individually screen CLBP participants who present to the physiotherapy centre. A limitation of this recruitment strategy is that we will not be capturing people from the community who do not seek 


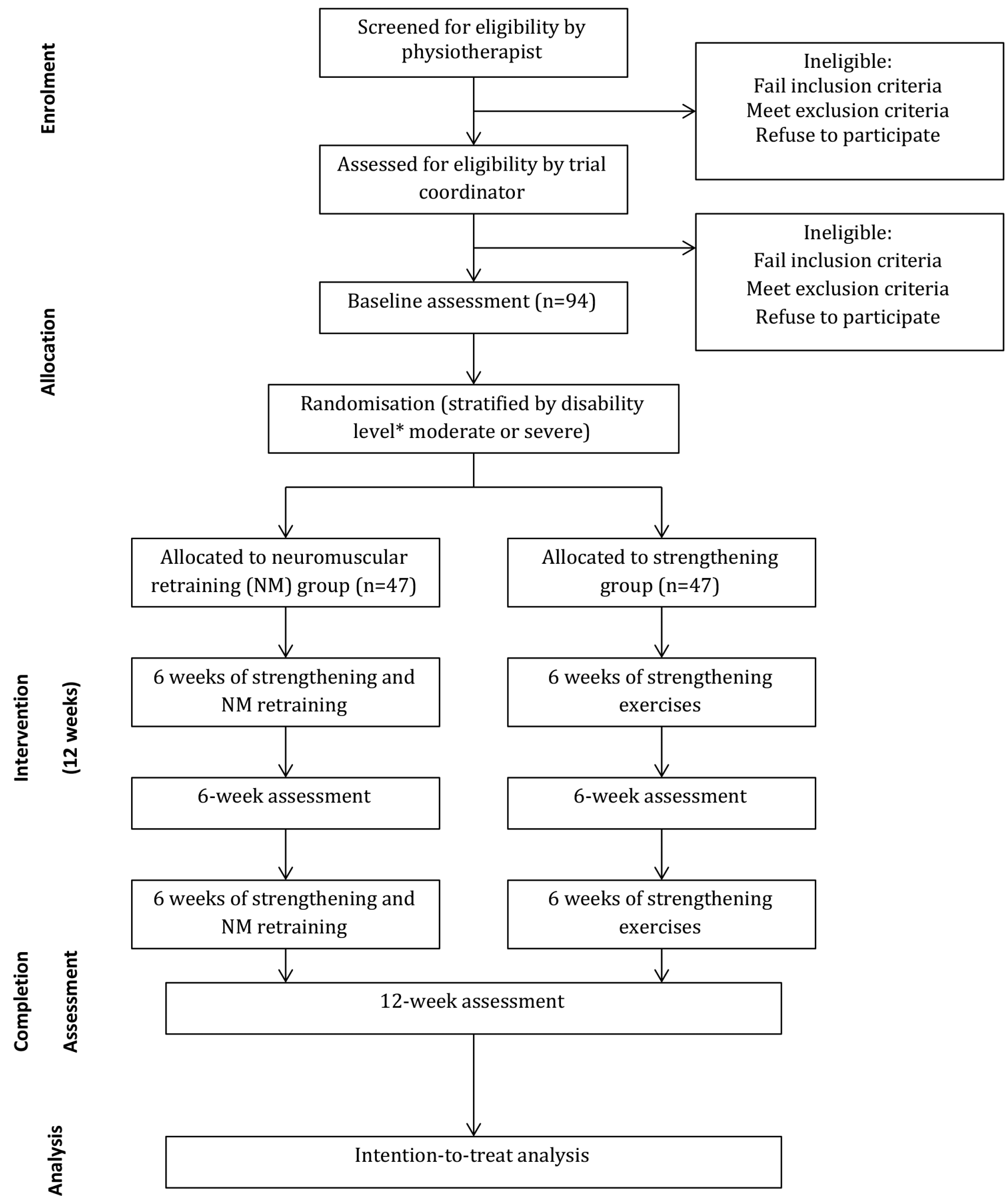

Figure 1 Study flow chart. *Disability is measured using the ODI; scores between $21 \%$ and $40 \%$ are classified as moderate disability and scores $\geq 41 \%$ are classified as severe disability.

physiotherapy treatment and may manage their back pain via other professionals or self-means. Participants who meet the eligibility criteria will be presented with written information about the study, including its procedures. Those interested in participating will provide written informed consent prior to randomisation. The inclusion and exclusion criteria are outlined in table 1 . Only participants with moderate or greater perceived disability (as per the ODI) will be included, as people with minimal CLBP-related disability have demonstrated similar results with a healthy population for the force accuracy assessment task. ${ }^{10}$ 
Table 1 Study inclusion and exclusion criteria

\begin{tabular}{|c|c|}
\hline Inclusion criteria & Exclusion criteria \\
\hline 1. Aged between 18 and 65 years & 1. Medication managed psychological illness \\
\hline $\begin{array}{l}\text { 2. Report chronic low back pain with or without pain } \\
\text { radiating into the lower limbs for at least } 3 \text { months }\end{array}$ & 2. Previous spinal and lower limb surgery \\
\hline
\end{tabular}

\section{Demonstrate moderate or greater disability on the 3. Diagnosed spinal osteoporosis/osteopaenia} ODI (ie, $21 \%$ or greater)

4. Diagnosed unstable spondylolisthesis/spondylolysis

5. Diagnosed active systemic/inflammatory joint disease

6. Diagnosed neurological and developmental disorders

7. Overt neurological sign (absence of lower limb reflex or motor paralysis)

8. Diagnosed significant medical conditions such as cancer or major cardiac diseases

9. History of abdo-pelvic organ prolapse

10.Use of medications that may influence balance

11.Patients funded by a compensable body

12.Inability to understand written/spoken English

13.Pregnant

14. $<6$ months postpartum

ODI, Oswestry Disability Index.

\section{INTERVENTIONS}

Participants from both treatment groups will participate in 12 weeks of machine-based resistance exercise sessions supervised in a one-to-one setting by qualified physiotherapists (1-5 years of experience). Each week, participants will attend two $30 \mathrm{~min}$ exercise sessions. The treating physiotherapists have been trained to deliver standardised interventions. All physiotherapists have undergone at least 15 hours of formal training in how to use the exercise machines (Delphex Kraeftigungstechnik). With respect to the novel neuromuscular control exercise, physiotherapists have undergone two one-to-one $30 \mathrm{~min}$ training sessions with the primary investigator (JBF) involved in the development of the exercise. On-site support will also be given to physiotherapists by one of the investigators (JBF) involved in the development of the exercise intervention.

During the 12-week programme, participants may also receive manual therapy and/or education by the treating physiotherapist. The type and dosage of co-interventions will be documented.

\section{Control group}

All participants enrolled in the RCT will undergo resistance training. Strengthening exercises will include a lumbar extension resistance exercise (figure 2A) together with one of the other resistance exercises (figure 2B-D) during each exercise session. The lumbar extension strength exercise is performed before any other strength exercise. Prior to completing the lumbar strength exercise, participants will complete a warm-up consisting of two lumbar extensions performed with no weight, as well

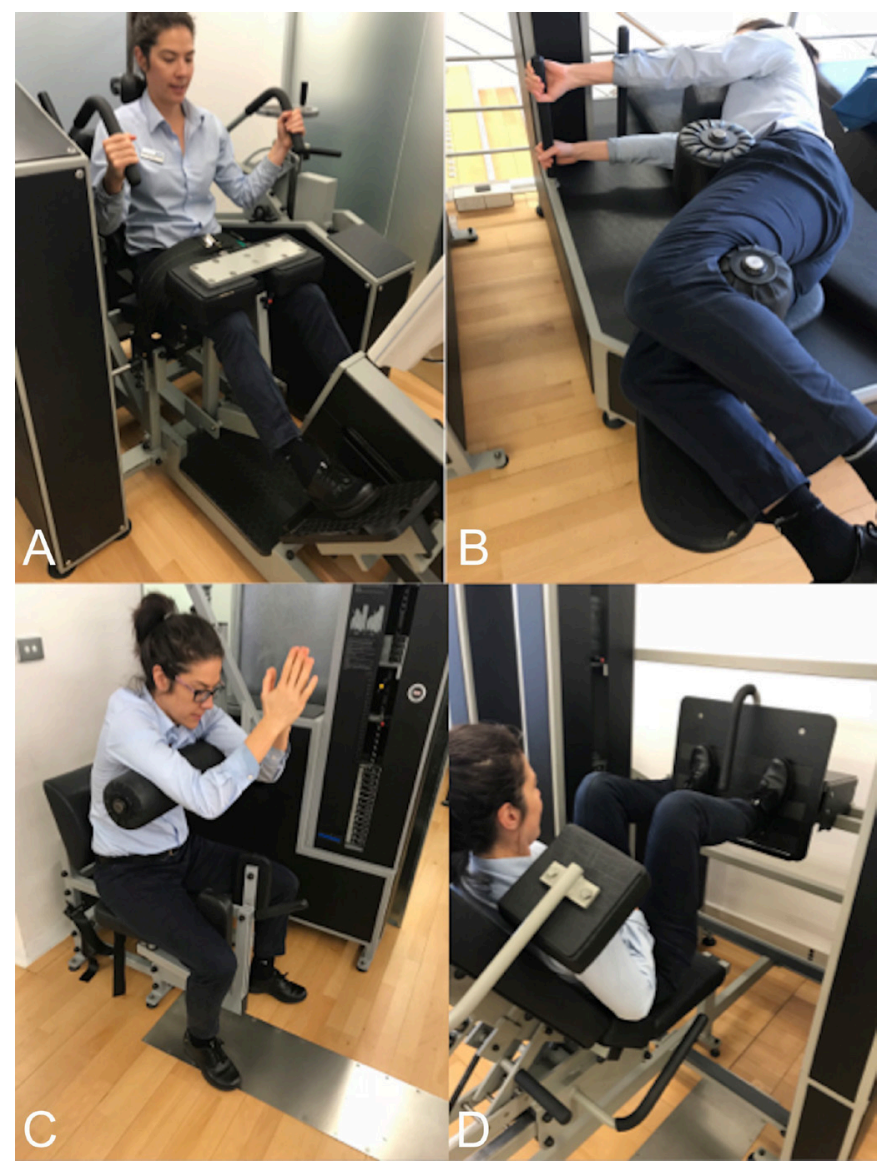

Figure 2 (A) Lumbar extension exercise. (B) Hip extension exercise. (C) Trunk flexion exercise. (D) Leg press exercise. 
Table 2 Training parameters and the progression of the force accuracy task for the entirety of the trial

\begin{tabular}{|c|c|c|c|c|c|}
\hline & Sets & Duration & Rest & Wave frequency & Force \\
\hline Weeks 1-4 & $\begin{array}{l}3 \text { (excluding the warm- } \\
\text { up) }\end{array}$ & $\begin{array}{l}60 \text { s for each } \\
\text { sinusoidal frequency }\end{array}$ & $\begin{array}{l}30 \text { s between } \\
\text { each set } \\
\text { (including the } \\
\text { warm-up) }\end{array}$ & $\begin{array}{l}\text { Slow }(0.05 \mathrm{~Hz}) \text {, medium } \\
(0.08 \mathrm{~Hz}) \text { and fast }(0.14 \mathrm{~Hz}) \\
\text { randomly ordered }\end{array}$ & $\begin{array}{l}20 \%-50 \% \text { of MVIC. } \\
\text { MVIC re-assessed at } \\
\text { the beginning of each } \\
\text { session }\end{array}$ \\
\hline Weeks 5-6 & $\begin{array}{l}3 \text { (excluding the warm- } \\
\text { up) }\end{array}$ & $\begin{array}{l}90 \text { s for each } \\
\text { sinusoidal frequency }\end{array}$ & $\begin{array}{l}30 \text { s between } \\
\text { each set } \\
\text { (including the } \\
\text { warm-up) }\end{array}$ & $\begin{array}{l}\text { Slow }(0.05 \mathrm{~Hz}) \text {, medium } \\
(0.08 \mathrm{~Hz}) \text { and fast }(0.14 \mathrm{~Hz}) \\
\text { randomly ordered }\end{array}$ & $\begin{array}{l}20 \%-50 \% \text { of MVIC. } \\
\text { MVIC re-assessed at } \\
\text { the beginning of each } \\
\text { session }\end{array}$ \\
\hline Weeks 7-8 & $\begin{array}{l}3 \text { (excluding the warm- } \\
\text { up) }\end{array}$ & $\begin{array}{l}120 \text { s for each } \\
\text { sinusoidal frequency }\end{array}$ & $\begin{array}{l}30 \text { s between } \\
\text { each set } \\
\text { (including the } \\
\text { warm-up) }\end{array}$ & $\begin{array}{l}\text { Slow }(0.05 \mathrm{~Hz}) \text {, medium } \\
(0.08 \mathrm{~Hz}) \text { and fast }(0.14 \mathrm{~Hz}) \\
\text { randomly ordered }\end{array}$ & $\begin{array}{l}20 \%-50 \% \text { of MVIC. } \\
\text { MVIC re-assessed at } \\
\text { the beginning of each } \\
\text { session }\end{array}$ \\
\hline Weeks 9-12 & $\begin{array}{l}3 \text { (excluding the warm- } \\
\text { up) }\end{array}$ & $120 s$ & $\begin{array}{l}30 \text { s between } \\
\text { each set } \\
\text { (including the } \\
\text { warm-up) }\end{array}$ & $\begin{array}{l}\text { Each set will contain a } \\
\text { random mixture of each } \\
\text { frequency (slow, medium, } \\
\text { fast) }\end{array}$ & $\begin{array}{l}20 \%-50 \% \text { of MVIC. } \\
\text { MVIC re-assessed at } \\
\text { the beginning of each } \\
\text { session }\end{array}$ \\
\hline
\end{tabular}

$\mathrm{Hz}$, hertz; MVIC, maximum voluntary isometric contraction.

as a 1 min set of the lumbar extension exercise (figure 2) at $50 \%$ of their training weight.

Participants in the control group will complete additional time on the lumbar extension exercise, at $50 \%$ of their maximum voluntary isometric contraction (MVIC), that is equivalent to the time (table 2) participants in the experimental group are performing the force accuracy exercise to control for work, that is if participants in the experimental group are completing 4 min of lumbar extensor force accuracy training, then the control participants will complete $4 \mathrm{~min}$ of lumbar extensor strength exercise performed at $50 \%$ of MVIC intensity.

Any other strengthening exercises (other than figure 2) prescribed to participants will be at the discretion of the supervising physiotherapist and will be documented in a training $\log$.

\section{Strength exercises image}

Participants will complete a MVIC on each machine they use throughout the study. The starting training weight will be set at $85 \%$ of the MVIC. Each strengthening exercise is to be completed for $90-120$ s with one-to-one supervision. Participants will perform continuous repetitions with a 3-s concentric and eccentric phase and a 2-s isometric phase throughout this time. Once the participant can complete the exercise for $120 \mathrm{~s}$, the physiotherapist will increase the resistance by $5 \%$ for the next session. This resistance training protocol conforms to the current American College of Sports Medicine guidelines. ${ }^{41}$ The treating therapist will assess participant's pain intensity before, during and after performing each exercise using the Numerical Rating Scale (NRS). If a participant's pain increases by a value of 3 out of 10 , then participants will cease the exercise.

\section{Experimental group}

In addition to resistance training, the intervention group will complete a neuromuscular control retraining exercise at the beginning of each training session. Specifically, participants will be seated on a MedX dynamometer (MedX) and will be instructed to push isometrically against the backrest with maximum voluntary effort. This MVIC data will be used to calculate the range of force required for the rest of the exercise. Participants will then be required to push back against the backrest to match a sinusoidal target force varying between $20 \%$ and $50 \%$ of their lumbar extension MVIC. ${ }^{10}$ The frequencies of the sinusoidal wave are $0.05 \mathrm{~Hz}, 0.08 \mathrm{~Hz}$ and $0.14 \mathrm{~Hz}$ (figure 3). Table 2 outlines the progression of the force accuracy exercise over the 12-week training programme.

Before beginning the force control exercise, all participants will complete a $1 \mathrm{~min}$ warm-up at a frequency of $0.08 \mathrm{~Hz}$ (medium). Following the warm-up, each session of force control exercise will involve participants completing three sets; each set will incorporate a different frequency (fast, medium or slow; see figure 3 ) that will be completed in a random order. A rest period of $30 \mathrm{~s}$ between each set of force control exercise will remain constant throughout the 12-week intervention.

\section{Outcome measures}

All primary, secondary and additional outcome measures will be assessed at baseline (prior to randomisation), after 6 weeks of treatment and at completion (12 weeks).

\section{Primary outcome}

Oswestry Disability Index (ODI)

The primary outcome of interest will be perceived functional disability and will be assessed using the ODI. ${ }^{42}$ The ODI comprises of a pain scale and nine categories pertaining to activities of daily living. Each component 

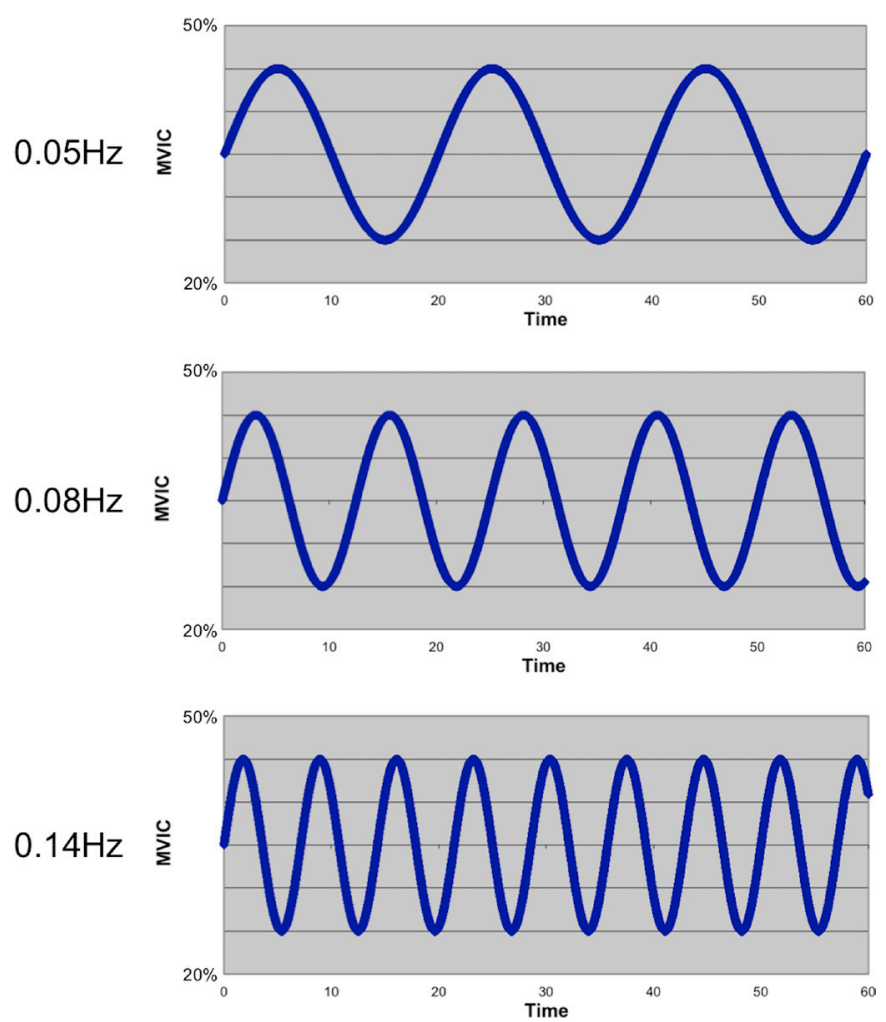

Figure 3 Sinusoidal waves for slow $(0.05 \mathrm{~Hz})$, medium $(0.08 \mathrm{~Hz})$ and fast $(0.14 \mathrm{~Hz})$ frequencies for the force accuracy assessment and training.

is scored from 0 to 5 . Overall scores are calculated as a percentage with higher values indicative of worse perceived disability. The ODI is reliable (intraclass correlation coefficient $($ ICC $)=0.94$ ), has good construct validity $(\mathrm{r}=0.99)^{43}$ and is sensitive to change in people with CLBP. ${ }^{44} 45$

\section{Secondary outcomes}

Numerical Rating Scale (NRS)

Pain severity will be recorded using the NRS. Participants will be asked to rate their average low back pain over the past week on an 11-point scale ranging from 0 (no pain) to 10 (worst imaginable pain). The NRS has demonstrated good construct validity and reliability in people with CLBP. ${ }^{46} 47$

\section{Tampa Scale for Kinesiophobia (TSK-17)}

The TSK-17 aims to measure pain-related fear beliefs about movement and re-injury ${ }^{48}$; it is scored using 17 questions about fear of pain and/or movement. Scores range from 17 to 68 ; higher scores indicate greater fear of movement. The TSK-17 has been demonstrated to have good construct validity and be a reliable (Cronbach's $\alpha=0.81)^{49} 50$ measure for pain-related fear and pain catastrophising in a CLBP cohort.

\section{Pain Self-Efficacy Questionnaire (PSEQ)}

The PSEQ asks 10 questions regarding pain and physical activity; Scores range from 0 to 60 ; lower scores indicate less confidence in recovering from CLBP. The PSEQ has good construct validity ${ }^{4}$ and is a reliable $(\mathrm{ICC}=0.92)^{51}$ measure in people with CLBP. It is also a useful tool to explain variability in perceived disability in this population. ${ }^{33}$

\section{International Physical Activity Questionnaire (IPAQ) Short Form}

The IPAQ Short Form will be used to evaluate participant's physical activity levels. Participants will provide information on their week's vigorous and moderate physical activity as well as walking time to calculate their metabolic equivalent in exercise testing. The IPAQ Short Form has demonstrated good reliability $(\mathrm{ICC}=0.80) .{ }^{52}$

\section{Additional outcomes}

A series of additional outcomes will be collected for the purposes of answering related questions about biomechanical effects of force accuracy and strength training, and for subsequent analyses of potential mediating effects on primary and secondary outcomes. These measures will be used to determine treatment efficacy.

\section{Lumbar extension strength}

Assessment of isometric lumbar extension strength using the MedX dynamometer has demonstrated good construct validity ${ }^{53}$ and reliability $(\mathrm{r}=0.57-0.93)^{54}$ in people with CLBP.

Participants will be seated on the MedX dynamometer (figure 4) with their trunk in $12^{\circ}$ flexion and lower limbs fixed. Using their lumbar extensor muscles, participants will then be required to push into the back force detection pad placed at the level of their thoracic spine with maximum voluntary effort. ${ }^{556}$ The force exerted on the load cell is measured as torque using custom developed LabView software. ${ }^{10}$

\section{Lumbar extension force accuracy}

Force accuracy assessment quantifies a person's ability to control and accurately produce sub-maximal, isometric force into a lumbar extension direction. This assessment has been demonstrated to be reliable $(\mathrm{ICC}=0.88)$ and have good construct validity in people with CLBP. ${ }^{10}$

While seated on the MedX dynamometer (as per earlier description), participants will then be asked to isometrically push against the backrest to match a fluctuating force trace (minimum and maximum thresholds of $20 \%$ MVIC and 50\% MVIC, respectively) displayed in real time on a computer tablet (figure 5). The sub-maximal range is based on lumbar extensor activity during daily tasks such as pushing a shopping cart. ${ }^{57}$

Participants will perform this force accuracy task at $0.08 \mathrm{~Hz}$ (medium speed) for $60 \mathrm{~s}$ as a warm-up. Participants will then perform three, 60-s assessments at three different frequencies in this order: (i) $0.08 \mathrm{~Hz}$ (five waves per minute; as per the warm-up), (ii) $0.05 \mathrm{~Hz}$ (three waves per minute; slow speed) and (iii) $0.14 \mathrm{~Hz}$ (8.4 waves per minute; fast speed). Participants will receive 30-s rest between the warm-up and each of the three assessments at different frequencies. 


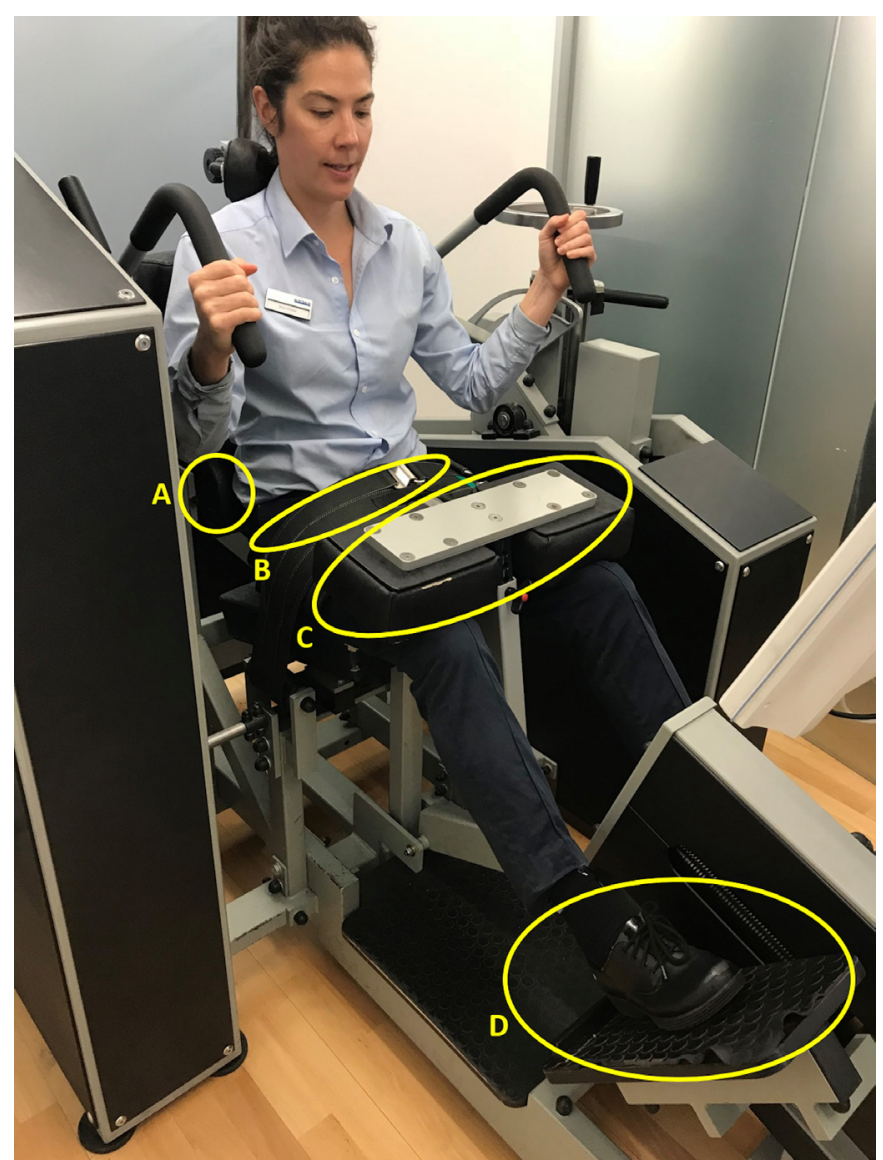

Figure 4 A participant seated on the MedX machine at $12^{\circ}$ lumbar flexion. The MedX restraint system includes the pelvic restraint $(A)$, lap belt $(B)$, thigh restraint $(C)$ and foot plate (D).

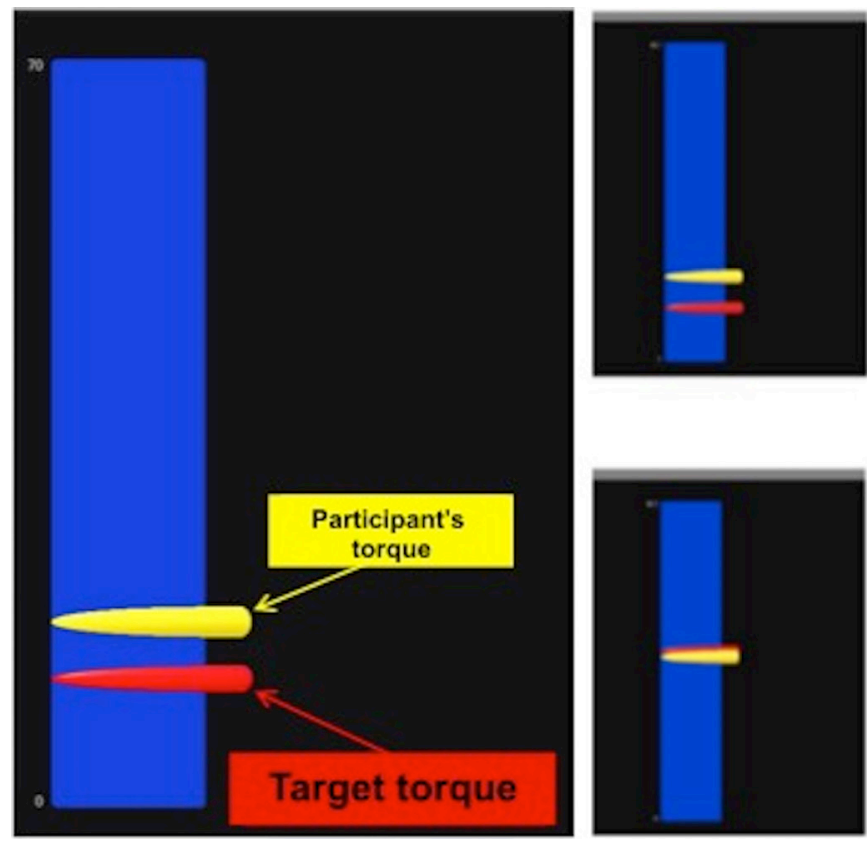

Figure 5 A screenshot of the muscle force accuracy assessment process as seen on the tablet by the participants. The red marker will move up and down at a speed depending on the frequency while the participant attempts to match this with the real-time yellow force marker. Reproduced with permission.
Lifting kinematics and kinetics

Kinetic and kinematic assessment during lifting has been demonstrated to be reliable ( $\mathrm{ICC}=0.98$ ) and has good construct validity for people with CLBP. ${ }^{11}$

For kinematic and kinetic analyses, participants will be assessed barefoot while wearing shorts. Non-reflective markers will be placed on the skin overlying specific vertebrae, as well as the head, pelvis, upper and lower limbs. Balance and force distribution through each lower limb will be assessed using Nintendo Wii Balance Boards (WBB, Nintendo) while three-dimensional mapping of anatomical landmarks will be performed using a 12-camera optoelectric motion analysis system (Optitrack Flex 13, NaturalPoint) sampling at $120 \mathrm{~Hz}$. Data from the WBBs will be collected on a laptop computer running custom software via a wireless Bluetooth connection. The WBB have demonstrated good construct validity against the appropriate gold-standard, laboratory-grade force platform. ${ }^{58}$ The Optitrack system has demonstrated good accuracy and reliability compared with the more expensive and widely used Vicon $612 .^{59}$

Participants will start the test standing upright with arms by their sides. An 8-kg weight was selected for functional relevance as this has been defined as the average weight of a bag of groceries. ${ }^{60}$ They will be given a standardised instruction to bend down and lift the weight with both hands from the ground to the level of their abdomen using a self-selected technique. They will then turn to their left and place the weight on a table at the height of their greater trochanter. ${ }^{12}$ They will then be instructed to place the weight back on the floor using a self-selected technique. The same assessment will also be performed on the right side. Four lifting trials will be conducted per side, the first serves as a practice trial. The average of the three remaining trials will be used for data analysis (total of 8 trials).

The kinematic data will be labelled, cleaned and gap-filled using Optitrack Motive software (NaturalPoint) and then passed through a custom written analysis pipeline (Visual3D v5.01.6, C-Motion). Angular displacement and angular velocity data will then be derived using custom written LabVIEW 2009 (National Instruments) software.

Ultrasonography of lumbar multifidus (LM) and iliocostalis lumborum (IL) muscles

Ultrasonography is a non-invasive reliable means of assessing muscle quantity and quality (including echogenicity ${ }^{25}$ and has been used in CLBP populations. ${ }^{9} 12256162$ Within this study, participants will lie in a prone position on a plinth with a pillow placed under their pelvis to avoid excessive lumbar lordosis. Images will be acquired using a Sonosite Turbo Edge machine (SonoSite, Fujifilm) with a linear transducer (6-13 MHz). To ensure consistency and reproducibility, bony landmarks including the spinous and transverse processes of L3, 4 and 5 will be palpated and marked using a water-soluble marker. Detection of the spinous processes was determined manually using the 
iliac crests as landmark guides. This has been previously demonstrated to be a reliable method for obtaining LM images. ${ }^{63}$ Transverse images of the LM will be taken adjacent to the spinous processes on both sides. Transverse images of the IL will be taken in line with the L3 spinous process on both sides. Images of the LM will be collected while resting and also during active contraction of the muscles. Active contraction images will be procured as participants perform a prone ipsilateral straight leg extension without bending their knee to the point where their knee is off of the plinth. To ensure consistency between test sessions, the angle of the participant's thigh relative to horizontal will be measured using a smart phone running inclinometer software (iHandy Level, V.1.70.3, iHandy) attached to the participant's thigh.

All US images will be measured using Image J software (NIH). Measures of muscle thickness will be determined by measuring the distance between the superior fascial border and the inferior fascial border of the muscle at the thickest point. Echogenicity will be measured via a circumferential trace of the inner fascial border of the muscle. The resting transverse images of the LM and IL will be measured for thickness and echogenicity, whereas the active images of the LM will only be measured for thickness. All measurements will be performed three times and an average will be calculated post image acquisition. For each side, the mean difference in LM thickness between the resting and contracted state will be compared.

\section{Sample size calculation}

The aim is to detect a 10-point difference in the primary outcome (ie, ODI), which corresponds to the minimal clinically important difference ${ }^{64}$ With a SD of 15 , this difference corresponds to an effect size of 0.67 , and the between-group ODI minimal clinical important difference can be detected with $80 \%$ power and a significance level of 0.05 with 37 participants per group. Allowing for $20 \%$ dropout, recruitment of $47(=37 /(1-0.2))$ participants per group is required.

\section{Randomisation and allocation concealment}

Following baseline assessment, participants will be randomised to one of the two treatment arms, using a computer-generated randomisation sequence, consisting of random permuted blocks of sizes $6-12$, stratified by baseline ODI level (moderate or severe/greater). The schedule will be stored on a password-protected website (REDCap, Vanderbilt University) maintained by an off-site co-investigator not involved in either participant recruitment or administration of primary/secondary outcome measures. This same co-investigator will reveal group allocation to the treating physiotherapists.

\section{Blinding}

Participants will be blinded to group allocation by the process of limited disclosure. Participants will not be informed about the exercises to be completed by participants in the other group. In addition, participants will not be informed about the study hypotheses, or which group they were allocated to, until study completion at which time they will be provided a summary of the study purpose, hypotheses and findings. The primary investigator will be conducting all the assessments and statistical analyses (in consultation with a biostatistician) and will not be involved in the random allocation process or treatment delivery and therefore, will not be privy to which group participants are allocated. As the primary and secondary outcomes are participant-reported, and participants are blinded, as well as the assessor conducting the biomechanical assessments is blinded, this study is also considered assessor-blinded. The statistician assisting with statistical analyses will not be involved in treatment delivery or assessment, nor with the randomisation process directly. A researcher not involved in the assessment, treatment or statistical analysis of participants will randomise and inform the treating physiotherapists, who are not blinded to the participant's group allocation.

\section{Data management}

All study data will be stored at the University of Melbourne for 15 years after the completion of the trial. All paperbased documents and data will be stored in a locked filing cabinet. All electronic data will be secured on a password-protected laptop and network drive. All documents that contain names or personal identifying information will be stored separately from other study data and identified by code number. Access to files will be limited to research staff involved in the study.

\section{Statistical analysis}

The principal investigator will analyse blinded data under the supervision of a biostatistician. Main comparative analyses between groups will be performed using intention-totreat. If the proportion of missing outcome data is greater than $5 \%$, multiple imputations will be used to account for missing outcome data. Data missing at baseline will be imputed using single mean imputation. Complete-case analyses will also be conducted. For the primary hypothesis, differences in mean change in disability index (baseline minus follow-up) will be compared between groups using linear regression modelling adjusted for baseline values and the stratifying variable of ODI level. Similar analyses will be conducted for continuous secondary outcomes. Standard diagnostic plots will be used to check the model assumptions of linearity and of constant variance and normality of residuals. All statistical analyses will be performed using SPSS V.21.0 (IBM) with significance level set at 0.05 . A sensitivity analysis will be conducted in the case where one group receives more co-interventions than the other group.

\section{Adverse events}

The neuromuscular control exercise has been piloted to minimise risk of pain exacerbation. While this novel neuromuscular control exercise has not been previously 
used for training people with CLBP, a similar protocol for assessment has been used in this population with no reports of pain exacerbation. ${ }^{10}$ The strengthening exercises and equipment utilised in this study have been previously used in people 2 weeks post lumbar discectomy. ${ }^{65}$ Of the 80 participants recruited, two dropped out due to increased pain with training. The risk of pain exacerbation is likely to be less, as this study does not recruit acute postoperative patients. In addition, previous studies and the American College of Sport Medicine recommendations ${ }^{4156}$ have used/prescribed similar intervention protocols, in similar settings and cohorts. An RCT investigating exercises of similar muscle groups reported a symptom exacerbation rate of $5.7 \% .^{66}$ The treatment protocols in this study involve constant close monitoring of participants.

Physiotherapists and the primary investigator will record any adverse events. Participants will also be asked to contact the primary investigator at their discretion by phone or email to report adverse events. If an adverse event is reported, the primary investigator, treating physiotherapists will liaise with local health service providers (eg, patients general practitioner (GP) or paramedics). All adverse events will be documented in the final written report of this study.

\section{Auditing}

A member of the research team not involved in determining eligibility, assessment or treatment of participants will perform fidelity checks to ensure the assessment and treatment of participants throughout the trial conforms to this protocol. Fidelity checks will be conducted every 3 months to ensure strict adherence. These checks will include observation of clinician's treatment of participants, hand-checks of the training logs describing the exercises completed for each session as well as online database (REDCap, Vanderbilt University) checks for assessment data storage. Breaches of protocol will be reported and further training to the assessor or therapist involved will be provided. A follow-up check of the breaching party will be conducted 2 weeks later to ensure the protocol is strictly adhered to.

\section{Ethical considerations}

Any amendment requiring modifications to the protocol, which may impact on the study, will be agreed on by the research team and will be submitted to the ethics committee for approval. Minor changes to the protocol will be agreed on by the research team and will be documented in a memorandum. All changes will be documented with the relevant trial registry.

Written informed consent will be obtained from all participants enrolled in the study. Participants will be informed that they have no obligation to participate in the study and are free to withdraw at any time, without any negative consequences on their future care. Participant's names and personal information will remain confidential at all times. This will be achieved by identifying data by the participant code and not using identifiable information; any identifiable information will be kept separate from study data.

\section{Patient and public involvement}

Patient priorities heavily impacted our decision to primarily investigate perceived disability in people with CLBP. While there are other mechanistic outcomes, understanding the impact that the interventions are having on a meaningful outcome for patients was paramount.

Patients were involved when developing and piloting the study assessment and intervention protocols. Patient's feedback regarding duration, difficulty and pain levels informed the protocol.

Patients presenting for treatment to a Melbourne-based physiotherapy clinic will be recruited for the proposed RCT. Trial participants will be able to obtain anonymous overall study results as well as their individual results from the trial after the completion of the study.

\section{DISSEMINATION}

Results of the RCT will be published in peer-reviewed journals. Data may be made available on request to the primary investigator, post-publication of all study results. The results of the trial will also be disseminated via conference presentations, professional organisations, media, social media and consumer organisations.

\section{CONCLUSION}

This will be the first RCT to compare the effects of neuromuscular control training with strength exercises on CLBP-related disability. The study results will provide valuable information about the physical and psychosocial mechanisms behind improvements in disability for people with CLBP.

\section{Author affiliations}

${ }^{1}$ Physiotherapy, School of Health Sciences, The University of Melbourne, Melbourne, Victoria, Australia

${ }^{2}$ Health Professions, Swinburne University of Technology, Hawthorn, Victoria, Australia

${ }^{3}$ Epidemiology and Preventative Medicine, Monash University, Melbourne, Victoria, Australia

Acknowledgements We would like to acknowledge Dr Ross Clark for his work developing the lifting and force accuracy assessment collection and analysis software, Dr Ben Mentiplay for his work assisting with the lifting kinematic data analysis, Dr Ben Metcalf for his work developing the randomisation schedule and implementation software, Professor Rana Hinman for her advice developing a double-blinded RCT and the patients and staff at Kieser Brighton for their involvement piloting the intervention.

Contributors JBF is a musculoskeletal physiotherapist who is undertaking his $\mathrm{PhD}$. $\mathrm{He}$ is leading the randomised controlled trial (RCT), managing budget, managing clinicians involved in the intervention, performing assessments and responsible for outcomes. He is also responsible for publishing the trial protocol and the findings of the trial. AP has assisted with the development of data collection and intervention protocols and data analysis of lumbar extensor force control, lifting movement coordination kinematics and kinetics. He has also assisted with the development of the intervention protocol, participant randomisation, trial fidelity assessment 
and manuscript preparation. GW has contributed to all aspects of RCT planning and implementation and publication, specifically helping to develop the novel force accuracy training protocol. DE-A has assisted with the study design, in particular the rationale and measurement protocol for ultrasound. She has also contributed to all aspects of the RCT planning, data analysis and publication. SMP has contributed to all aspects of RCT planning and publication, specifically developing the measurement protocol using ultrasound. JK has assisted with the sample size calculation and formulated data analysis plan. JK has mentored the corresponding author, JBF, in analysis and statistical calculations at the completion of the trial. $A B$ has contributed to all aspects of RCT planning, implementation and publication. He has assisted with the development of data collection and intervention protocols and data analysis of lumbar extensor force control, lifting movement coordination kinematics and kinetics.

Funding The authors have not declared a specific grant for this research from any funding agency in the public, commercial or not-for-profit sectors.

Competing interests None declared.

Patient consent for publication Not required.

Ethics approval This study was approved by the University of Melbourne Behavioural and Social Sciences Human Ethics Sub-Committee on 8 August 2017, reference number 1749845 .

Provenance and peer review Not commissioned; externally peer reviewed.

Open access This is an open access article distributed in accordance with the Creative Commons Attribution Non Commercial (CC BY-NC 4.0) license, which permits others to distribute, remix, adapt, build upon this work non-commercially, and license their derivative works on different terms, provided the original work is properly cited, appropriate credit is given, any changes made indicated, and the use is non-commercial. See: http://creativecommons.org/licenses/by-nc/4.0/.

\section{REFERENCES}

1. Hoy D, March L, Brooks $P$, et al. The global burden of low back pain: estimates from the global burden of disease 2010 study. Ann Rheum Dis 2014;73:968-74.

2. Hartvigsen J, Hancock MJ, Kongsted A, et al. What low back pain is and why we need to pay attention. The Lancet 2018;391:2356-67.

3. Vos T, Allen C, Arora M, et al. Global, regional, and national incidence, prevalence, and years lived with disability for 310 diseases and injuries, 1990-2015: a systematic analysis for the global burden of disease study 2015. The Lancet 2016;388:1545-602.

4. Chiarotto A, Deyo RA, Terwee CB, et al. Core outcome domains for clinical trials in non-specific low back pain. Eur Spine $J$ 2015;24:1127-42.

5. Chapman JR, Norvell DC, Hermsmeyer JT, et al. Evaluating common outcomes for measuring treatment success for chronic low back pain. Spine 2011;36(21 Suppl):S54-S68.

6. Clement RC, Welander A, Stowell C, et al. A proposed set of metrics for standardized outcome reporting in the management of low back pain. Acta Orthop 2015;86:523-33.

7. Mannion AF, Junge A, Taimela S, et al. Active therapy for chronic low back pain: Part 3. factors influencing self-rated disability and its change following therapy. Spine 2001;26:920-9.

8. Steele J, Bruce-Low S, Smith D. A reappraisal of the deconditioning hypothesis in low back pain: review of evidence from a triumvirate of research methods on specific lumbar extensor deconditioning. Curr Med Res Opin 2014;30:865-911.

9. Hides J, Gilmore C, Stanton W, et al. Multifidus size and symmetry among chronic LBP and healthy asymptomatic subjects. Man Ther 2008;13:43-9.

10. Pranata A, Perraton L, El-Ansary D, et al. Lumbar extensor muscle force control is associated with disability in people with chronic low back pain. Clin Biomech 2017;46:46-51.

11. Pranata A, Perraton L, El-Ansary D, et al. Trunk and lower limb coordination during lifting in people with and without chronic low back pain. J Biomech 2018;71:257-63.

12. Sánchez-Zuriaga D, López-Pascual J, Garrido-Jaén D, et al. Reliability and validity of a new objective tool for low back pain functional assessment. Spine 2011;36:1279-88.

13. Coenen P, Gouttebarge V, van der Burght ASAM, et al. The effect of lifting during work on low back pain: a health impact assessment based on a meta-analysis. Occup Environ Med 2014;71:871-7.

14. Nelson JM, Walmsley RP, Stevenson JM. Relative lumbar and pelvic motion during loaded spinal flexion/extension. Spine 1995;20:199-204.

15. Mokhtarinia HR, Sanjari MA, Chehrehrazi M, et al. Trunk coordination in healthy and chronic nonspecific low back pain subjects during repetitive flexion-extension tasks: effects of movement asymmetry, velocity and load. Hum Mov Sci 2016;45:182-92.

16. Marras WS. The working back: a systems view. Hoboken, NJ: WileyInterscience, 2008.

17. Hides J, Stanton W, Mendis MD, et al. The relationship of transversus abdominis and lumbar multifidus clinical muscle tests in patients with chronic low back pain. Man Ther 2011;16:573-7.

18. Rezazadeh F, Taheri N, Okhravi SM, et al. The relationship between cross-sectional area of multifidus muscle and disability index in patients with chronic non-specific low back pain. Musculoskelet Sci Pract 2019;42:1-5.

19. Danneels LA, Vanderstraeten GG, Cambier DC, et al. A functional subdivision of hip, abdominal, and back muscles during asymmetric lifting. Spine 2001;26:E114-E121.

20. Panjabi MM. The stabilizing system of the spine. Part I. function, dysfunction, adaptation, and enhancement. J Spinal Disord 1992;5:383-9.

21. Danneels LA, Vanderstraeten GG, Cambier DC, et al. CT imaging of trunk muscles in chronic low back pain patients and healthy control subjects. Eur Spine J 2000;9:266-72.

22. Barker KL, Shamley DR, Jackson D. Changes in the cross-sectional area of multifidus and psoas in patients with unilateral back pain: the relationship to pain and disability. Spine 2004;29:E515-9.

23. Kader DF, Wardlaw D, Smith FW. Correlation between the MRI changes in the lumbar multifidus muscles and leg pain. Clin Radiol 2000;55:145-9.

24. Wan $Q$, Lin C, Li X, et al. MRI assessment of paraspinal muscles in patients with acute and chronic unilateral low back pain. $\mathrm{Br} J$ Radiol 2015;88:20140546.

25. Stokes M, Rankin G, Newham DJ. Ultrasound imaging of lumbar multifidus muscle: normal reference ranges for measurements and practical guidance on the technique. Man Ther 2005;10): :116-26.

26. Cholewicki J, VanVliet IV JJ. Relative contribution of trunk muscles to the stability of the lumbar spine during isometric exertions. Clinical Biomechanics 2002;17:99-105.

27. Larivière $C$, Gagnon $D$, Loisel $P$. The comparison of trunk muscles EMG activation between subjects with and without chronic low back pain during flexion-extension and lateral bending tasks. $J$ Electromyogr Kinesiol 2000;10:79-91.

28. Lee $\mathrm{H}$, Hong $\mathrm{JH}$. Comparison of trunk muscle activities in lifting and lowering tasks at various heights. J Phys Ther Sci 2016;28:585-8.

29. Buchbinder R, van Tulder M, Öberg B, et al. Low back pain: a call for action. The Lancet 2018;391:2384-8.

30. Salvetti MdeG, Pimenta CAdeM, Braga PE, et al. [Disability related to chronic low back pain: prevalence and associated factors]. Rev EsC Enferm USP 2012;46 Spec No:16-23.

31. Picavet HSJ, Vlaeyen JWS, Schouten JSAG. Pain catastrophizing and kinesiophobia: predictors of chronic low back pain. Am J Epidemiol 2002;156:1028-34.

32. de Moraes Vieira EB, de Góes Salvetti M, Damiani LP, et al. Self-Efficacy and fear avoidance beliefs in chronic low back pain patients: coexistence and associated factors. Pain Manag Nurs 2014;15:593-602.

33. Costa LdaCM, Maher CG, McAuley JH, et al. Self-Efficacy is more important than fear of movement in mediating the relationship between pain and disability in chronic low back pain. Eur $J$ Pain 2011;15:213-9.

34. Vibe Fersum K, O'Sullivan P, Skouen JS, et al. Efficacy of classification-based cognitive functional therapy in patients with nonspecific chronic low back pain: a randomized controlled trial. Eur J Pain 2013;17:916-28.

35. Campbell P, Foster NE, Thomas E, et al. Prognostic indicators of low back pain in primary care: five-year prospective study. J Pain 2013;14:873-83.

36. Hayden J, van Tulder MW, Malmivaara A, et al. Exercise therapy for treatment of non-specific low back pain. Cochrane Database Syst Rev 2005;35.

37. Saragiotto BT, Maher CG, Yamato TP, et al. Motor control exercise for chronic non-specific low-back pain. Cochrane Database Syst Rev 2016;21.

38. Wang X-Q, Zheng J-J, Yu Z-W, et al. A meta-analysis of core stability exercise versus general exercise for chronic low back pain. PLoS One 2012; 7:e52082

39. Ferreira ML, Ferreira PH, Latimer J, et al. Comparison of general exercise, motor control exercise and spinal manipulative therapy for chronic low back pain: a randomized trial. Pain 2007;131:31-7.

40. Chang $W-D$, Lin $H-Y$, Lai $P-T$. Core strength training for patients with chronic low back pain. J Phys Ther Sci 2015;27:619-22.

41. Garber CE, Blissmer B, Deschenes MR, et al. American College of sports medicine position stand. quantity and quality of exercise for developing and maintaining cardiorespiratory, musculoskeletal, 
and neuromotor fitness in apparently healthy adults: guidance for prescribing exercise. Med Sci Sports Exerc 2011;43:1334-59.

42. Fairbank JC, Pynsent PB. The Oswestry disability index. Spine 2000;25:2940-53.

43. Davidson M, Keating JL. A comparison of five low back disability questionnaires: reliability and responsiveness. Phys Ther 2002;82:8-24.

44. Davidson M. Rasch analysis of three versions of the Oswestry disability questionnaire. Man Ther 2008;13:222-31.

45. Monticone M, Baiardi P, Vanti C, et al. Responsiveness of the Oswestry disability index and the Roland Morris disability questionnaire in Italian subjects with sub-acute and chronic low back pain. Eur Spine J 2012;21:122-9.

46. Pengel LHM, Refshauge KM, Maher CG. Responsiveness of pain, disability, and physical impairment outcomes in patients with low back pain. Spine 2004;29:879-83.

47. Jamison RN, Raymond SA, Slawsby EA, et al. Pain assessment in patients with low back pain: comparison of Weekly recall and momentary electronic data. J Pain 2006;7:192-9.

48. Kori SH, Miller RP, Todd DD. Kinesiophobia: a new view of chronic pain behavior. Pain Management 1990;3:35-43.

49. Roelofs J, Goubert L, Peters ML, et al. The Tampa scale for Kinesiophobia: further examination of psychometric properties in patients with chronic low back pain and fibromyalgia. Eur J Pain 2004;8:495-502.

50. Goubert L, Crombez G, Van Damme S, et al. Confirmatory factor analysis of the Tampa scale for Kinesiophobia: invariant two-factor model across low back pain patients and fibromyalgia patients. Clin J Pain 2004;20:103-10.

51. Maughan EF, Lewis JS. Outcome measures in chronic low back pain Eur Spine J 2010;19:1484-94.

52. Craig CL, Marshall AL, Sjöström M, et al. International physical activity questionnaire: 12 -country reliability and validity. Med Sci Sports Exerc 2003;35:1381-95

53. Pollock ML, Graves JE, Leggett SH. Accuracy of counter weighting to account for upper body mass in testing lumbar extension strength. Med Sci Sports Exerc 1991;23.

54. Robinson ME, Greene AF, O'Connor P, et al. Reliability of lumbar isometric torque in patients with chronic low back pain. Phys Ther 1992;72:186-90.

55. Graves JE, Webb DC, Pollock ML, et al. Pelvic stabilization during resistance training: its effect on the development of lumbar extension strength. Arch Phys Med Rehabil 1994;75:210-5.
56. Steele J, Bruce-Low S, Smith D, et al. A randomized controlled trial of limited range of motion lumbar extension exercise in chronic low back pain. Spine 2013;38:1245-52.

57. Lee Y-J, Hoozemans MJM, van Dieën JH. Trunk muscle control in response to (un)expected turns in cart pushing. Gait Posture 2012;36:133-8.

58. Clark RA, Bryant AL, Pua Y, et al. Validity and reliability of the Nintendo Wii balance board for assessment of standing balance. Gait Posture 2010;31:307-10.

59. Carse B, Meadows B, Bowers R, et al. Affordable clinical gait analysis: an assessment of the marker tracking accuracy of a new low-cost optical 3D motion analysis system. Physiotherapy 2013;99:347-51.

60. Silvetti A, Mari S, Ranavolo A, et al. Kinematic and electromyographic assessment of manual handling on a supermarket green- grocery shelf. Work 2015;51:261-71.

61. Hides JA, Stokes MJ, Saide M, et al. Evidence of lumbar multifidus muscle wasting ipsilateral to symptoms in patients with acute/ subacute low back pain. Spine 1994;19:165-72

62. Hides JA, Richardson CA, Jull GA. Magnetic resonance imaging and ultrasonography of the lumbar multifidus muscle. Comparison of two different modalities. Spine 1995;20:54-8.

63. Wallwork TL, Hides JA, Stanton WR. Intrarater and interrater reliability of assessment of lumbar multifidus muscle thickness using rehabilitative ultrasound imaging. J Orthop Sports Phys Ther 2007;37:608-12.

64. Ostelo RWJG, Deyo RA, Stratford P, et al. Interpreting change scores for pain and functional status in low back pain: towards international consensus regarding minimal important change. Spine 2008;33:90-4.

65. Choi G, Raiturker PP, Kim M-J, et al. The effect of early isolated lumbar extension exercise program for patients with herniated disc undergoing lumbar discectomy. Neurosurgery 2005;57:764-72.

66. Manniche C, Lundberg E, Christensen I, et al. Intensive dynamic back exercises for chronic low back pain: a clinical trial. Pain 1991;47:53-63.

67. Deyo RA, Dworkin SF, Amtmann D, et al. Report of the NIH Task force on research standards for chronic low back pain. Pain Med 2014;15:1249-67.

68. Walker BF. The prevalence of low back pain: a systematic review of the literature from 1966 to 1998. J Spinal Disord 2000;13:205-17. 